\section{Versorgung diabetischer Füße: Telemedizin im Fokus}

\author{
Patienten mit Diabetes mellitus und Fußulcera benötigen ein qualitativ hoch- \\ wertiges Management, was besonders in Flächenregionen eine große \\ Herausforderung ist. Hier könnte die Telemedizin helfen. Welche Faktoren \\ sind bei Etablierung der modernen Technologie wichtig? Dieser Frage ging \\ ein norwegisches Forscherteam in einer qualitativen Studie nach.
}

A n der Untersuchung zur Versorgung von Patienten mit Diabetes mellitus und Fußulcera nahmen 34 medizinische Mitarbeiter teil. Die meisten von ihnen waren Krankenpfleger ( $n=29)$, von denen fünf eine Führungsposition innehatten. Unter den verbleibenden fünf Teilnehmern fanden sich je zwei Podologen und Ärzte sowie ein Pflegehelfer. Insgesamt 21 der Probanden arbeiteten in der Primärversorgung. Die Autoren führten zehn Fokusgruppen-Interviews mit drei bis sieben Teilnehmern durch. Die Wissenschaftler trennten die Gruppen nach medizinischem Fachpersonal der Primär- und der Spezialversorgung, das Führungspersonal traf sich separat.

Die qualitative Untersuchung war Teil einer randomisierten Studie, in der die Auswirkungen einer interaktiven Telemedizin-Plattform untersucht werden sollte. Für die aktuelle Arbeit fokussierten die Autoren die Versorgung von $\mathrm{Pa}$ tienten mit diabetischen Fußulcera mittels der Telemedizin-Technologie. Diese schloss eine webbasierte Ulkus-Krankenakte und via Mobiltelefon versendete Fotos sowie Berichte zu den Ulcera von dem primärmedizinisch Tätigen an das spezialisierte Personal ein. Die Patienten wurden zu Hause, in Kliniken der Erstversorgung und in allgemeinärztlichen Praxen untersucht. Im Abstand von sechs Wochen suchten sie die Fachambulanz der spezialisierten Kliniken auf.

Nach Auswertung der Interviews wurden vier Punkte identifiziert, die für eine erfolgreiche Implementierung der Telemedizin-Plattform notwendig waren:

- Nutzerfreundliche Technologie und

Schulungen zum Einsatz dieser _Ein Telemedizin-Experte im Team

- Engagiertes und verantwortliches

Führungspersonal

_Effektive Kommunikationskanäle im

organisatorischen Bereich
Insgesamt schätzten die Teilnehmer die verwendete Technologie als einfach im Umgang ein. Für sie war es wichtig, dass ihre Meinung bei technischen Anpassungen berücksichtigt wurde. Ein Telemedizin-Experte im Team oder in erreichbarer Nähe erschien den Probanden als essenziell für den Erfolg der Telemedizin. Dabei war es für die spezialisierten Fachkräfte weitaus wahrscheinlicher, einen Telemedizin-Experten im Team zu haben, als für die primärmedizinischen Fachkräfte.

Hinsichtlich des engagierten und verantwortlichen Führungspersonals zeigten sich ebenfalls Unterschiede zwischen den beiden medizinisch tätigen Gruppen. Während sich das Personal der Fachabteilungen durch ihre Vorgesetzten gut unterstützt fühlte, fühlte sich das primärversorgende Personal eher auf sich gestellt. Bezüglich effektiver Kommunikationskanäle gaben Führungskräfte im primärmedizinischen Bereich an, dass sie die Ansprechpartner in den Spezialabteilungen kennen müssen. Für das primärversorgende Personal stellte es sich als ein Problem heraus, dass sie oft nur allein oder zu zweit an der Intervention teilnahmen. Das spezialisierte Personal sah es dagegen als Vorteil an, nur mit wenigen Personen kommunizieren zu müssen.

Die Ergebnisse zeigen, wie wichtig es ist, die unterschiedlichen Bedürfnisse des medizinischen Personals in verschiedenen Arbeitsbereichen zu berücksichtigen. Zwar sind die Resultate aus qualitativen Studien nicht generalisierbar, doch könnten sie bei der Identifizierung von Hindernissen und unterstützenden Faktoren helfen, so die Autoren. (aks) roducing telemedicine in diabetes foot care: a qualitative inquiry. BMC Nurs. 2017;16:2
Kolltveit BH et al.: Conditions for success in int-

\section{KOMMENTAR}

Die Studie von Kolltveit et al. zeigt, dass der Einsatz von Telemedizin den Informationsaustausch zwar unterstützen kann, gleichzeitig aber auf eine funktionierende Interaktion zwischen den Beteiligten angewiesen ist. Bereits bei der Entwicklung der eingesetzten Software trägt die Fähigkeit der Beteiligten zur gegenseitigen Verständigung dazu bei, die Technologie bedarfsgerecht und nutzerfreundlich zu gestalten. Im ständigen Austausch über die Bedürfnisse der Anwender mit den Technologieexperten nähern sich zwei völlig verschiedene Fachbereiche an und schaffen ein effizientes Hilfsmittel für den gezielten Informationsaustausch. Was Softwareentwickler und Anwender bei Kolltveit et al. geschafft haben, ist auch zwischen medizinischen Spezialisten und Primärversorgern essentiell. Insbesondere die in der Primärversorgung benannten Schwierigkeiten (z.B. fehlende Ansprechpartner) und die Sorge, wichtige Informationen zu verpassen, deuten darauf hin, dass es bei der Zusammenarbeit zwischen Spezialisten und Basispersonal noch Entwicklungsbedarf in der interdisziplinären Kommunikation gibt.

Der Hinweis, dass die Fortführung der telemedizinischen Betreuungsprozesse in der Primärversorgung durch Abwesenheiten der wenigen Verantwortlichen gefährdet ist, zeigt, dass selbst innerhalb eines Teams Übergaben nicht sicher gewährleistet sind. Der Einsatz von Telemedizin kann deshalb nur soweit erfolgreich sein, wie das tägliche Bemühen um gelingende Kommunikation zwischen allen Beteiligten. Ein erfolgreicher Einsatz setzt somit vernetztes Denken in dem Sinne voraus, dass alle Beteiligten den Informationsbedarf der anderen Netzwerkpartner mitdenken und aktiv den Austausch suchen. Im Fokus steht die Verständigung zur Erreichung eines gemeinsamen Zieles - Telemedizin ist hier hilfreich, aber nicht ausreichend.

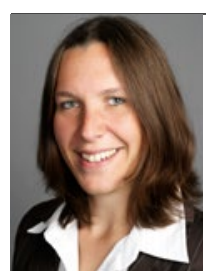

Katja Hodeck IGM GmbH / DiabetesPflege-Akademie Berlin Allee der Kosmonauten 33g, 10318 Berlin 